\title{
Schreiben des Herrn d'Abbadie an den Herausgeber.
}

La lunetre zénitale est le seul appareil astronomique qui n'exige, pour ainsi dire, aucune correction instrumentale. Il est donc bien à regretter qu' aucun astronome he s'en serve, à l'exception de M. Airy, dont l'infatigable activité se tient plutôt en avant des derniers progrès de l'astronomie, qu'à leur niveau seulement. La lunette zénitale de ce savant est la première solution pratique du probléme, car le projet antérieur de Mr. Faye n'a pas été mis à exécution. Ici comme partout on simplitie en perfectionnant, et l'instrument de $M$. Porro, qui est une lunette ordinaire surmontée d'un vase d'eau à fond transparent, se laisse transporter facilement et fait de la lunette zénitale un instrument géodésique. C'est en l'employant que nos officiers d'état major ont déterminé, en très-peu de jours, la latitude de Paris avec une précision comparable à tout ce que permettent les plus couteux instruments d'un grand observatoire. Il me semble que tout astronome devrait observer et puhlier sa zone zénitale; ce serait d'ailleurs l'un des moyens les plus précis d'étudier ces légères variations de la latitude sur lesquelles je travaille depuis plusieurs années et que M. Airy vient enfin de constater de son coté.

Quoiqu'il en soit, je viens proposer deux applications nouvelles de la lunette zénitale. Au commencement de ce Siècle on s'inquiétait beaucoup de l'inégalité d'obliquité des Solstices. On croyait à une différence de 7 ou 8 secondes et quelques astronomes ne sont pas encore persuadés que cette différence soit nulle. Or on résoudrait définitivement la question en s'établissant un peu en dédans de chaque tropique de manière à observer à son zénit la culmination de chaque bord du soleil, en ayant soin de la comparer micrométriquement, à peu d'heures d'intervalle, aux étoiles qui avoisinent le tropique. Cette ohservation pourrait de faire plus commodément en Amériqne, mais en Afrique on aurait la faculté, qui est peut-être un avantage, d'observer les deux solstices sous le mème méridien, par exemple, sous celui de Suez.

Mais l'autre usage de la lunette zénitale sera plus facilement réalisé. Je veux parler de la détermination de l'attraction des nontagnes. Pour cela on se pourvoirait de deux lunettes zénitales de M. Porro, qui ont l'avantage d'étre les moins coutenses et l'on observerait e $n$ mème temps les mémes étoiles zénitales au nord et au sud de la montagne. J'ai la confiance que des observations de ce genre faites an M: Schehallien en Ecosse, donneraient une attraction différente de 5"8, valeur trouvée par Maskelyne, qui la déduisait de différences d'apozénits, qui n'étaient pas obtenues avec les deux conditions si essentielles de simultanéité et d'absence de corrections instrumentales. Pour répondre à toutes les objections on devrait d'ailleurs faire une seconde suite de ces observations après avoir transporté à la station septentrionale la lunette zénitale déjà employée an sud de la montagne, et vice versa. J'ai lien de présumer en effet, d'après une longue suite d'observations de niveaux fixes, que la direction du fil à plomb peut varier d'un jour à l'autre, et que e'est là peut-ètre la cause de cette fluctuation des latitudes à laquelle je crois depuis longtemps.

Avant de terminer cettre lettre, permettez-moi d'appeler l'attention de vos lecteurs sur un sujet, qui interesse les amateurs d'étoiles doubles. Dans un mémoire de Mr. Klinkerfues sur l'orbite de $p$ Ophiuchi, publié dans le $\mathbf{X} 1135$ des Astr. Nachr., on a cité cinq suites d'observations conme étant de M. Bishop, tandis que les trois premières suites, datées $1841,67,1842,53$ et 1843,47 sont de M. Dawes, et les deux dernières sont de M. Hind. C'est le 18 janvier que M. Dawes quitta l'observatoire de M. Bishop et la dernière ohservation que M. Dawes y fit a pour date 1844,044. Antérieurement à cette date c'est au seul M. Dawes que sont dues toutes les observations publiés par M. Bishop, à la seule exception d'ane suite de mesures de 8 Lacertae, imprimée à la page 41 avec la remarque spéciale que cette ohservation a été faite par M. Bishop. Du reste cette volume mentionnc plus d'une fois comme faites par M. Daros des mesures notées un peu plus has avec le nom de M. Bishop à coté. Par exemple on peut comparer à cet égard la page 57 sous l'étoile 36 d'Andromède et la page 62 sous $y$ d'Andromède. --

11 est bon de signaler ces erreurs et d'appeler l'attention des calculateurs sur le vrai observateur de chaque suite de mesures, car l'équation personnelle peut jofier un role important dans ces évaluations si delicates.

Paris 1858 Aout $5 . \quad$ Antoine d'Abbadie.

\section{Schreiben des Herrn Dr. Förster an den Herausgeber.}

Wäbrend ich damit heschätigt bin, eine grössere Reihe von Planeteu-Beobachtungen zur Poblikation vorzubereiten, scheint es mir nirht übertlïssig, die folgenden einzelnen Beobachtungen Ihnen mitzutheilen, weil dieselben geeignet 